\title{
Detection of SARS-CoV-2 in the gastrointestinal tract among patients with negative nasopharyngeal COVID-19 testing prior to endoscopy
}

\section{(ㄷ)(1) $(-)$}

\author{
Authors \\ Dennis Yang1, Yaseen B. Perbtani ${ }^{1}$, Julia Loeb ${ }^{2,3}$, Nanlong Liu' ${ }^{1}$, Peter V. Draganov ${ }^{1}$, David E. Estores ${ }^{1}$, Michael \\ Lauzardo $^{3,4}$, Anthony Maurelli2,3, John A. Lednicky ${ }^{2,3}$, J. Glenn Morris ${ }^{3,4}$
}

Institutions

1 Division of Gastroenterology, Hepatology and Nutrition, University of Florida, Gainesville, Florida, United States

2 Department of Environmental and Global Health, College of Public Health and Health Professions, University of Florida, Gainesville, Florida, United States

3 Emerging Pathogens Institute, University of Florida, Gainesville, Florida, United States

4 Department of Medicine, College of Medicine, University of Florida, Gainesville, Florida, United States

submitted 1.2.2021

accepted after revision 31.3.2021

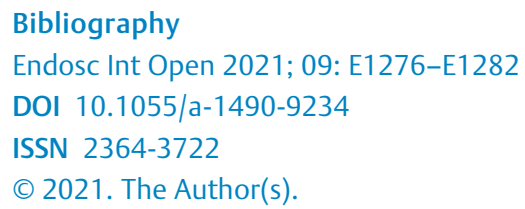

丹 Supplementary material is available under https://doi.org/10.1055/a-1490-9234

\section{ABSTRACT}

Background and study aims The clinical significance of SARS-CoV-2 RNA in the stool remains unclear. We aimed to determine whether SARS-CoV-2 is detected via real-time reverse transcriptase polymerase chain reaction ( $r R T-P C R$ ) in the gastrointestinal tracts of patients scheduled for endoscopy and if the virus obtained from these clinical specimens could be isolated in culture.

Patients and methods All patients underwent symptom screening and had negative nasopharyngeal testing for SARS-CoV-2 within 72 hours of their scheduled procedure. Study samples were collected via nasopharyngeal swab, rectal swab, and fluid from the upper gastrointestinal tract and/or colon based on their endoscopic procedure(s). Samples were tested for SARS-CoV-2 via rRT-PCR. SARS-CoV-2 positive specimens were isolated and cultured in Vero-E6 cells.

Results 243 patients (mean age 63.1 years;54.3\% men) were enrolled from July 15, 2020 to September 2, 2020. SARS-CoV-2 testing was performed from 242 (99.6\%) nasopharyngeal, 243 (100\%) rectal, 183 (75.3\%) upper gastrointestinal tract and $73(30 \%)$ colon samples. SARS-CoV-2 RNA was detected in the nasopharynx and gastrointestinal specimens in one patient $(0.4 \%)$. After a 14 -day incubation period, there was no evidence of virus growth in cells incubated with any of these specimens.

Conclusions SARS-CoV-2 was rarely detected in the gastrointestinal tract of patients with negative nasopharyngeal testing prior to endoscopy. No live virus was detected by culture, further highlighting that presence of viral genome on its own is not sufficient proof of infectivity. PCR-based screening provides limited insight into virus infectivity and its results should be interpreted carefully as to avoid unnecessary delays in clinical care or inadvertent risk exposure.

\section{Introduction}

The novel coronavirus, SARS-CoV-2, which has infected more than 75 million people and caused more than 1.6 million deaths worldwide, is primarily transmitted through respiratory secre- tions, droplets and direct contact [1-5]. Endoscopy has been regarded as high risk for infection transmission, given the demand for short physical distance between the endoscopy personnel and the patient, and the risk of generating aerosols and micro-droplets during the procedures. Several strategies have 
been introduced to mitigate the risk of infection transmission, with national gastrointestinal societies issuing joint guidance recommendations regarding endoscopy during the COVID-19 pandemic [6]. Among these, patients are recommended to undergo pre-procedure COVID-19 screening, performed by realtime reverse transcriptase polymerase chain reaction (rRTPCR) testing via nasopharyngeal swab.

There is a growing body of evidence indicating that the gastrointestinal (gastrointestinal) tract is targeted by SARS-CoV-2. A recent meta-analysis reported a pooled prevalence of $48.1 \%$ of SARS-CoV-2 in stool samples among COVID-19 patients, with $70.3 \%$ of these patients testing positive after respiratory samples were negative [7]. Notably, patients with viral shedding in the stool and no or mild respiratory symptoms and negative nasopharyngeal swabs would go undetected under the current standards of practice for pre-endoscopy COVID-19 screening. However, both the prevalence and clinical significance of virus positivity in the gastrointestinal tract remains unclear. This study aimed to prospectively determine: (1) whether SARS-CoV-2 is detected in the gastrointestinal tract of patients with negative routine nasopharyngeal COVID-19 testing prior to their scheduled endoscopy; and (2) if the virus obtained from these clinical specimens could be isolated in culture.

\section{Patients and methods}

This was a prospective study to determine whether SARS-CoV-2 was in the gastrointestinal tract of patients undergoing endoscopic procedures as part of their standard of care from July 15, 2020 to September 2, 2020. This study was approved by the UF Institutional Review Board (IRB202000488). All authors had access to the study data and reviewed and approved the final manuscript.

\section{Study participants and COVID-19 screening procedure}

All patients admitted to the endoscopy unit for a procedure(s) were eligible for study participation. Patients had already undergone routine testing for SARS-CoV-2 via rRT-PCR of nasopharyngeal swabs within 24 to 72 hours of their scheduled procedure as part of our institution's mandated policy. Signed research informed consent was obtained from all patients prior to enrollment.

\section{Sample collection for SARS-CoV-2}

Sample collection for SARS-CoV-2 was performed in the endoscopy room. Nasopharyngeal and rectal/ostomy swab sample collections were routinely performed on all subjects as part of the study. Additional sample collection from the gastrointestinal lumen (i. e. fluid from upper gastrointestinal tract or colon secretions) were obtained depending on the type of endoscopy the patient had been scheduled for as part of his/her routine care. All personnel wore personal protective equipment (PPE), which included N95 respiratory masks (or equivalent), gowns and eye protection [2].

Nasopharyngeal swab sample collection: The swab was inserted into the nostril and gently rotated inward until a depth equal to the distance from the nostrils to the outer opening of the ear was reached. The swab was then rotated for 5 to $10 \mathrm{sec}$ onds to absorb secretions [8]. Swabs were then placed in the viral transport media (VTM). The VTM consisted of a sterile screw-cap polypropylene tube containing $1 \mathrm{~mL}$ of a formulation of brain heart infusion broth-based VTM [9].

Rectal/ostomy swab sample collection: Sample collection was performed prior to insertion of the endoscope. The swab was inserted approximately 1 inch into the anal canal (or ostomy if applicable) and rotated while swabbing from side to side. The swab was allowed to remain approximately $10 \mathrm{sec}$ onds to absorb secretions prior to removal and then placed in the VTM.

Sample collection from the gastrointestinal tract: For sample collection in the gastrointestinal tract, the endoscope was inserted into the lumen and 5- to 20 - $\mathrm{mL}$ aliquots of luminal secretions were aspirating through the suction channel. The aspirate was collected into a designated specimen trap (Bard Medical; Covington, Georgia, United States) attached to the scope. Following collection, the swab was inserted into the specimen trap and rotated for approximately 10 seconds with the sample to absorb the secretions. Swab samples were then placed in the labeled VTM.

Sample handling: All specimens in their respectively labeled VTM tubes were inserted into ice at collection, maintained refrigerated at $2^{\circ} \mathrm{C}$ to $8^{\circ} \mathrm{C}$ thereafter, and transported (within 48 hours from the time of collection) to the SARS-CoV-2 research laboratory of JAL at the University of Florida (UF) Emerging Pathogens Institute (EPI).

\section{SARS-CoV-2 RNA purification and rRT-PCR}

In the biosafety level 2-enhanced laboratory, RNA was extracted from the nasopharyngeal, rectal/ostomy swabs, and upper gastrointestinal and colon fluid specimens and rRT-PCR performed as detailed in the supplemental material. Two rRT-PCR methods for the detection of the SARS-CoV-2 N-gene were used: the CDC N2 primer system $[9,10]$ and the Lednicky Ngene primer system ( $\vee$ Table $\mathbf{1}$ ). If the internally developed test and a CDC N2 primer test agreed, the sample was assigned the consensus result. If the tests disagreed, we ran an additional CDC N1 primer test to develop a consensus result.

\section{SARS-CoV-2 isolation and culture}

All procedures for viral culture followed strict laboratory biosafety guidelines and were conducted in a biosafety level 2 enhanced facility using biosafety level 3 working practices as detailed in the supplemental material. The confirmed rRT-PCR positive SARS-CoV-2 specimens were inoculated onto Vero E6 cells (American type Culture Collection [ATCC], Manassas, Virginia, United States). The cells were then maintained in a $37^{\circ} \mathrm{C}$ incubator over a 14-day period, with refeeds performed at 7-day intervals and daily observations of the cytopathic effect (CPE).

\section{Follow-up}

All patients were contacted within 72 hours (immediately if test-positive) with the results of their SARS-CoV-2 testing. Subjects with SARS-CoV-2 positivity from any of the collected spe- 
- Table 1 SARS-CoV-2 N-gene rRT-PCR primers and probes.

\begin{tabular}{|c|c|c|c|}
\hline $\begin{array}{l}\text { Primer and probe } \\
\text { name }\end{array}$ & Description & Oligonucleotide sequence & $\begin{array}{l}\text { Fluorophore, } \\
\text { Quencher }\end{array}$ \\
\hline Led-N-F & SARS CoV-2 N Forward Primer & 5'-GGGAGCAGAGGCGGCAGTCAAG-3' & None \\
\hline Led-N-R & SARS CoV-2 N Reverse Primer & 5'-CATCACCGCCATTGCCAGCCATTC-3' & None \\
\hline Led-N-Probe ${ }^{1}$ & SARS CoV-2 N Probe & FAM-CCTCATCACGTAGTCGCAACAGTTC-BHQ1 & FAM, BHQ1 \\
\hline 2019-nCov_N2-F & 2019-nCov_N2 Forward Primer & 5'-TTACAAACATTGGCCGCAAA-3' & None \\
\hline 2019-nCov_N2-R & 2019-nCov_N2 Reverse Primer & GCGCGACATTCCGAAGAA & None \\
\hline 2019-nCov_N2-P² & 2019-nCov_N2 Probe & FAM-ACAATTTGC /ZEN/ CCCCAGCGC TTCAG-3IABkF & FAM, ZEN, 3IABkFQ \\
\hline
\end{tabular}

cimens were considered to have COVID-19. Positive COVID-19 patients and their health care providers were alerted immediately, provided with infectious disease consultation at UF, and referred to the Department of Health in their respective county [11]. All positive patients were contacted weekly to assess for symptoms and any medical treatment received. Repeat testing for SARS-CoV-2 via nasopharyngeal and rectal swab was planned at 30-day follow-up for positive patients. All patients, irrespective of testing results, were contacted to assess for COVID19 related symptoms at 14 to 21 days following their procedure.

Data collection and statistical analysis: All data was prospectively recorded using a data collection form and then entered into a central electronic database. Data collection included patient demographics, indication and type of procedures, and COVID-19 related symptom questionnaire (Supplemental material). Descriptive statistics for each baseline variable was obtained and expressed as mean, standard deviation (SD), median and interquartile ranges (IQR).

\section{Results}

\section{Study population and baseline characteristics}

A total of 967 patients underwent endoscopic procedures in our unit from July 15, 2020 to September 2, 2020. Of them, 243 patients provided research consent and were enrolled in the study. All study subjects had negative tests for SARS-CoV2 on nasopharyngeal swab on routine pre-endoscopy screening within 72 hours prior to their scheduled procedure. Tw of the 243 patients $(0.82 \%)$ had prior documented COVID-19 illness. In these two patients, COVID-19 was diagnosed 45 and 15 days prior to their scheduled endoscopy. Both patients had symptomatic resolution with negative testing for SARS-CoV-2 from two sequential nasopharyngeal specimens collected $\geq 24$ hours apart, in addition to the third negative nasopharyngeal swab performed as part of the mandatory pre-endoscopy screening $\leq 72$ hours prior to their procedures.

Patient and procedural characteristics are summarized in - Table 2. Most patients (177; 72.8\%) reported no COVID-19 related symptoms, with nausea/vomiting (23; 9.5\%), cough $(17 ; 7 \%)$ and fatigue $(17 ; 7 \%)$ being the three most commonly endorsed among symptomatic patients. The two most common indications for endoscopy were abdominal pain $(58 ; 23.9 \%)$ and dysphagia (45; $18.5 \%)$. Indications and type of endoscopic procedures are summarized in > Table 2.

\section{Sample collection for SARS-CoV-2}

Among the 243 subjects included in the study, sample collection for SARS-CoV-2 testing was obtained from nasopharyngeal swab in 242 (99.6\%), rectal/ostomy swab in 243 (100\%), from the upper gastrointestinal tract in 183 (75.3\%) and from the colon in 73 (30\%). One patient declined having a specimen collected from the nasopharynx. All collected specimens were adequate for rRT-PCR testing except for insufficient material from one rectal and one upper gastrointestinal tract sample from two different patients. Both patients tested negative for all their remaining samples collected from the other sites.

\section{SARS-CoV-2 rRT-PCR test results}

One patient tested positive for SARS-CoV-2 in all her clinical samples (nasopharynx, ostomy swab, upper gastrointestinal tract and colon). The patient was a 65-year-old woman with an end colostomy due to a history of multiple abdominal surgeries complicated by a colocutaneous fistula. She had been scheduled for elective upper endoscopy and colonoscopy by her gastroenterologist for evaluation of chronic diarrhea. Forty-five days prior to her procedures, the patient had presented with a 3-day history of fever, intractable nausea, vomiting, and headaches and tested positive for SARS-CoV-2 on RT-PCR from a nasopharyngeal swab sample. She had symptom resolution within 7 days of onset followed by two sequential negative nasopharyngeal specimens on days 5 and 8 after onset of illness. She also had a third negative nasopharyngeal swab test 72 hours prior to endoscopy. The patient and her health care provider were contacted within 24 hours after she tested positive for SARS-CoV-2 in the study. The patient denied any COVID-19-related symptoms up to 26 days after her testing. She declined further follow-up or repeat testing as part of the study. 


\begin{tabular}{|c|c|}
\hline Mean age (interquartile range), years & $63.2(55-73)$ \\
\hline Male/Female & $132 / 111$ \\
\hline \multicolumn{2}{|l|}{ Race; n (\%) } \\
\hline - Caucasian & $203(83.5)$ \\
\hline - African American & $33(13.6)$ \\
\hline - Asian & $4(1.6)$ \\
\hline - Other & $2(0.8)$ \\
\hline \multicolumn{2}{|l|}{ Comorbidities; n (\%) } \\
\hline - Seasonal allergies & $110(45.3)$ \\
\hline - Diabetes mellitus & $57(23.5)$ \\
\hline - Hypertension & $118(48.6)$ \\
\hline - Chronic obstructive pulmonary disease/asthma & $33(13.6)$ \\
\hline - Coronary artery disease & $57(23.5)$ \\
\hline - Chronic liver disease & $24(9.9)$ \\
\hline - Chronic kidney disease & $20(8.2)$ \\
\hline - Inflammatory bowel disease & $4(1.6)$ \\
\hline - Cancer & $7(2.9)$ \\
\hline - Tobacco use & $39(16)$ \\
\hline \multicolumn{2}{|l|}{ COVID-19 related symptoms; n (\%) } \\
\hline - Fever & $10(4.1)$ \\
\hline - Cough & $17(7)$ \\
\hline - Fatigue & $17(7)$ \\
\hline - Shortness of breath & $15(6.2)$ \\
\hline - Sputum production & $9(3.7)$ \\
\hline - Nasal congestion & $6(2.5)$ \\
\hline - Sore throat & 0 \\
\hline - Headache & $1(0.4)$ \\
\hline " Joint/muscle ache & $5(2.1)$ \\
\hline " Nausea or vomiting & $23(9.5)$ \\
\hline " Diarrhea & $14(5.8)$ \\
\hline - Loss of taste or smell & 0 \\
\hline \multicolumn{2}{|l|}{ Demographics; n (\%) } \\
\hline - Florida resident & $237(97.5)$ \\
\hline - Florida non-resident & $6(2.5)$ \\
\hline \multicolumn{2}{|l|}{ Travel outside of the United States in last 14 days; $n$ (\%) } \\
\hline - Yes & $3(1.2)$ \\
\hline - No & $240(98.8)$ \\
\hline \multicolumn{2}{|l|}{ Indications for endoscopic procedure; n (\%) } \\
\hline - Dysphagia & $45(18.5)$ \\
\hline - Nausea/vomiting & $18(7.4)$ \\
\hline - Abdominal pain & $58(23.9)$ \\
\hline
\end{tabular}

\begin{tabular}{|c|c|}
\hline \multicolumn{2}{|l|}{ - Table $\mathbf{2}$ (Continuation) } \\
\hline - Change in bowel habits & $25(10.3)$ \\
\hline - Bloating/gas & $16(6.6)$ \\
\hline - Blood in stool & $20(8.2)$ \\
\hline - Bowel obstruction & $7(2.9)$ \\
\hline - Pancreaticobiliary intervention & $32(13.2)$ \\
\hline - Tumor staging & $8(3.3)$ \\
\hline - Surveillance/treatment of Barrett's esophagus & $14(5.8)$ \\
\hline $\begin{array}{l}\text { - Endoscopic mucosal resection/endoscopic sub- } \\
\text { mucosal dissection }\end{array}$ & $34(14)$ \\
\hline - Surveillance after polyp removal & $13(5.3)$ \\
\hline - Colorectal cancer screening & $6(2.5)$ \\
\hline \multicolumn{2}{|l|}{ Type of endoscopy; n (\%) } \\
\hline - Esophagoduodenoscopy (EGD) & $126(45.3)$ \\
\hline - Upper endoscopic ultrasound (EUS) & $36(12.9)$ \\
\hline $\begin{array}{l}\text { - Endoscopic retrograde cholangiopancreatogra- } \\
\text { phy (ERCP) }\end{array}$ & $40(14.4)$ \\
\hline - Small bowel enteroscopy & $9(3.2)$ \\
\hline - Colonoscopy & $65(23.4)$ \\
\hline - Lower endoscopic ultrasound (EUS) & $2(0.7)$ \\
\hline \multicolumn{2}{|l|}{ COVID-19 testing site; $\mathrm{n}(\%)$} \\
\hline - Nasopharynx & $242(99.6)$ \\
\hline - Upper gastrointestinal tract secretions & $183(75.3)$ \\
\hline - Lower gastrointestinal tract secretions & $73(30.0)$ \\
\hline - Rectal swab & $243(100)$ \\
\hline
\end{tabular}

\section{SARS-CoV-2 rRT-PCR in COVID-19 patient}

The rRT-PCR CDC N2 cycle quantification values $\left(C_{q}\right)$ for each sample collection site in the COVID-19 patient are presented in $>$ Table 3 , with the corresponding instrument output shown in $>$ Fig. 1 . The $C_{q}$ value represents the PCR cycle number at which the sample's curve intersected the threshold line. The sample from the colon had the highest $C_{q}$, followed by the one obtained from the nasopharyngeal swab, upper gastrointestinal tract, and rectal/ostomy swab.

\section{SARS-CoV-2 culture-based isolation}

Virus culture was attempted on all the SARS-CoV-2 positive specimens isolated from the COVID-19 patient. After a 14-day incubation period, there was no evidence of virus growth in the Vero E6 cells incubated with the four different specimens (nasopharyngeal, upper gastrointestinal tract, colon, and ostomy isolates). 
- Table 3 CDC N2 rRT-PCR test results for positive case.

\begin{tabular}{|c|c|c|}
\hline Specimens & rRT-PCR test & CDC N2 $C_{q}$ Value \\
\hline Positive control ${ }^{1}$ & + & 28.81 \\
\hline Negative control ${ }^{2}$ & - & 0 \\
\hline Nasopharyngeal swab & + & 34.64 \\
\hline Ostomy swab & + & 35.04 \\
\hline $\begin{array}{l}\text { Upper gastrointestinal } \\
\text { tract fluid }\end{array}$ & + & 34.68 \\
\hline Colon fluid & + & 29.71 \\
\hline \multicolumn{3}{|c|}{$\begin{array}{l}\text { CDC, Centers for Disease Control and Prevention; RT-PCR, reverse tran- } \\
\text { scriptase polymerase chain reaction } \\
{ }^{1} \text { SARS-COV-2 N-gene plasmid. } \\
{ }^{2} \text { No template control. }\end{array}$} \\
\hline
\end{tabular}

\section{Follow-up}

Out of the 242 patients with negative testing, 197 (81.4\%) were reached within 14 to 21 days of their procedure. None of these patients endorsed any COVID-19 related symptoms nor had tested positive for SARS-CoV-2. Twenty of 35 inpatients in this study had repeat SARS-CoV-2 testing during their hospitalization; all of which were negative.

\section{Discussion}

The COVID-19 pandemic has spread worldwide at an alarming pace, causing a global health crisis. Initial efforts to mitigate the risk of infection transmission in the endoscopy unit involved delaying all elective endoluminal procedures. As new COVID-19 diagnosis rates have begun to plateau, multiple gastrointestinal societies have attempted to issue guidance on how to safely resume endoscopic services $[2,12,13]$. However, much uncertainty remains, given the limited availability of data to support decision-making. There has been ongoing concern for the potential of fecal-oral transmission of SARS-CoV-2 during endoscopy given its persistence in fecal samples among COVID-19 patients $[7,14]$. However, in this prospective study, we demonstrated that in an area of intermediate disease prevalence, SARS-CoV-2 RNA was rarely detected in the gastrointestinal tract of patients with negative COVID-19 screening via nasopharyngeal swab prior to endoscopy. Importantly, even when SARS-CoV-2 RNA was isolated from respiratory and gastrointestinal specimens, infectious virus was not detected by cell culture. Our results further emphasize that the presence of viral genome on its own is not sufficient proof of infectivity, and calls for a word of caution when interpreting positive preendoscopy PCR-based results.

In the guidance for endoscopic resumption during the current pandemic, multiple gastrointestinal societies have recommended routine pre-endoscopy testing for SARS-CoV-2 via nasopharyngeal swab sampling $[2,12,13]$. Three prior retrospective reports evaluating the implementation of universal preprocedure testing demonstrated that the positivity for COVID19 among asymptomatic patients scheduled for endoscopy is

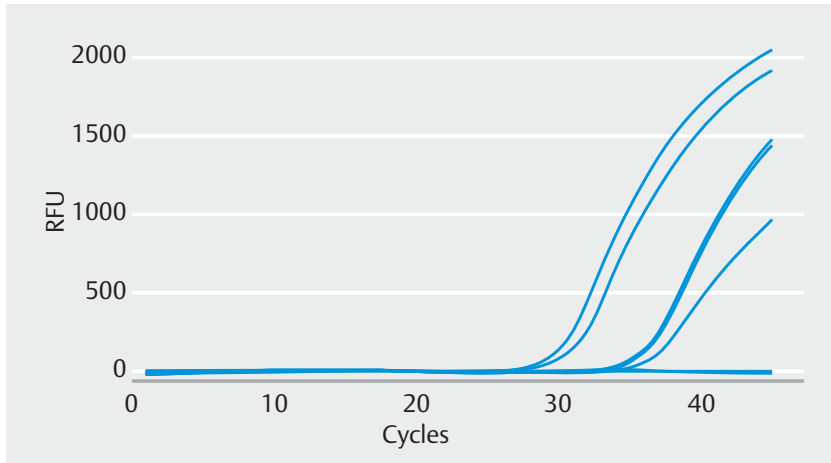

- Fig. 1 rRT-PCR CDC N2 cycle quantification values $\left(C_{q}\right)$ for each sample collection site in the COVID-19 patient are presented in - Table 3, with the corresponding instrument output shown in - Fig. 1. The $C_{\mathrm{q}}$ value represents the PCR cycle number at which the sample's curve intersected the threshold line.

rare, ranging between $0.2 \%$ to $1 \%$ in areas of low to intermediate disease prevalence [15-17]. Similarly, during our study period, the cumulative percent positive in our county was $6.14 \%$ [18], yet only one patient $(1 / 243 ; 0.4 \%)$ tested positive for SARS-CoV-2. Our findings were comparable to infection rates observed in patients screened prior to endoscopic procedures across various academic and community-based endoscopy centers in the country $[15,16]$. The overall lower than expected positivity rate among patients suggest the potential efficacy of screening questionnaires in identifying those individuals at high risk. Yet, as shown in our study, gastrointestinal manifestations of COVID-19 [7,14] often overlap with common indications for endoscopy. In all, asymptomatic COVID-19 patients or those with gastrointestinal manifestations but no or mild respiratory symptoms remain a screening dilemma.

Universal SARS-CoV-2 testing potentially allows the identification of COVID-19 patients that may be otherwise missed with symptom screening alone. However, current PCR testing of nasopharyngeal samples has been associated with significant variability in performance $[19,20]$. In our study, the patient who tested positive for SARS-CoV-2 in all of her collected clinical specimens had an initial negative nasopharyngeal swab PCRbased test. These findings further underscore the limitations related to the diagnostic performance of current assays and the potential implications of either false negative or positive results on patient care and safety. Future improvement and standardization of testing assays are urgently needed.

Serial viral RNA testing in COVID-19 patients have shown persistent stool positivity in $70.3 \%$ of individuals despite negative respiratory samples and irrespective of symptoms, raising concern of fecal-oral transmission by these patients who would be otherwise missed under the current guidance recommendations for COVID-19 screening prior to endoscopy [7,20-24]. Reassuringly, our study demonstrated that the overall prevalence of patients with virus positivity in the gastrointestinal tract with negative nasopharyngeal samples on pre-procedure testing was very low $(0.4 \%)$, occurring in only one patient who had prior documented COVID-19 illness. Noteworthy, while 
PCR has become a widely accepted diagnostic strategy given its wide application and rapid performance, it should be emphasized that PCR performs poorly in detecting replicative virus and thereby provides limited insight into virus infectivity [25, 26]. In our study, none of the SARS-CoV-2-positive specimens by rRT-PCR showed evidence of viral growth after a 14-day incubation period, further alluding to the fact that RT-PCR positivity may persist significantly beyond infectivity. Our data are consistent with those previously reported by Wölfel and colleagues, who demonstrated that no live virus could be isolated from stool collected 8 days after symptom onset, in spite of persistently high viral RNA loads in these samples [27-32]. The significance of viral RNA detection in the gastrointestinal tract and the possibility of oral-fecal transmission remains unknown, as live virus isolation from the gastrointestinal tract, has not been demonstrated [27]. Future prospective well-designed studies aiming to evaluate the temporal association between disease onset and viral infectivity in the gastrointestinal tract are needed.

There are several strengths to our study. Most of the reports on SARS-CoV-2 in stool samples from COVID-19 patients have been retrospective and from China. To our knowledge, this is the first prospective study from the West systematically and simultaneously collecting samples for SARS-CoV-2 testing from the nasopharynx and various gastrointestinal sites. Importantly, our estimation of viral infectivity in the gastrointestinal tract was based on virus isolation and culture studies, which provides superior data with regards to viral replication as compared to estimates based on viral load alone as detected by PCR [32].

We also acknowledge the limitations of this study. This was a single-center study at a large tertiary care center where all patients underwent routine COVID-19 testing prior to endoscopy; therefore, our results may not be generalizable to all endoscopy centers worldwide. The lower than predicted rate of SARS-CoV2 RNA positivity in the gastrointestinal tract in this study may have been due to selection bias, as study subjects had a negative nasopharyngeal COVID-19 screening test prior to their scheduled procedure. Furthermore, we recognize that the percent positive of COVID-19 reported in this study could have been influenced by the relatively small sample size and may not representative of other communities with different disease prevalence. However, the low positivity rates from this study are in concordance with the similarly low rates reported among endoscopy centers in areas with different disease prevalence [15-17]. Importantly, it should be noted that the results from this study are also dependent on testing characteristics, which have yet to be rigorously evaluated and validated for SARS-CoV2 detection. Lastly, our results should be interpreted with caution due to the very small sample size of patients with SARSCoV-2 positivity in the gastrointestinal tract identified in this study. Future studies with larger sample sizes are needed to further determine the clinical impact of virus presence in the gastrointestinal tract.

\section{Conclusions}

In summary, SARS-CoV-2 was rarely detected in the gastrointestinal tract of patients with routine negative nasopharyngeal PCR testing prior to endoscopy in an area of intermediate disease prevalence. None of the SARS-CoV-2-positive clinical specimens showed viral replicability in culture. Our data suggest that the presence of viral RNA does not imply infectivity and interpretation of PCR testing should always be performed within the clinical context, so as to mitigate unnecessary delays in patient care or inadvertent risk exposure to staff and other patients. Future studies are needed to better define the implications of SARS-CoV-2 in the gastrointestinal tract and its transmissibility risk.

\section{Acknowledgements}

This study was supported by internal funding from the University of Florida Emerging Pathogens Institute and the Clinical and Translational Science Institute. The authors thank Tatiana Ramirez-Hiller, Research Coordinator of the Division of Infectious Diseases and Global Health, and April Goddard and Becky Brown, Research Coordinators of Division of Gastroenterology and Hepatology, for their contributions to this study.

\section{Competing interests}

Dr. Yang is a consultant for Boston Scientific, Lumendi, and Steris. Dr. Draganov is a consultant for Cook Medical, Olympus, Boston Scientific, Lumendi, and Microtech.

\section{References}

[1] Johns Hopkins Coronavirus Resource Center. https://coronavirus.jhu. edu/map.html [Accessed December 19th, 2020]

[2] Sultan S, Lim JK, Altayer O et al. AGA Institute Rapid Recommendations for Gastrointestinal Procedures During the COVID-19 Pandemic. Gastroenterology 2020; 159: 739-758.e4

[3] European Centre for Disease Prevention and Control - Latest evidence on COVID-19. https://www.ecdc.europa.eu/en/covid-19/latest-evidence [Accessed September 28th, 2020]

[4] World Health Organization (WHO). Q\&A: How is COVID-19 transmitted? https://www.who.int/news-room/q-a-detail/q-a-how-is-covid19-transmitted [Accessed September 28th, 2020]

[5] He X, Lau EHY, Wu P et al. Temporal dynamics in viral shedding and transmissibility of COVID-19. Nat Med 2020; 26: 672-675

[6] AGA/DHPA joint guidance for resuming elective endoscopy during COVID-19 pandemic. https://gastro.org/news/aga-dhpa-release-guidance-for-resuming-elective-endoscopy Accessed August 26th, 2020

[7] Cheung KS, Hung IFN, Chan PPY et al. Gastrointestinal manifestations of SARS-CoV-2 infection and virus load in fecal samples from a Hong Kong cohort: systematic review and meta-analysis. Gastroenterology 2020; 159: 81-95

[8] Centers for Disease Control and Prevention (CDC) guidelines for clinical specimen collection. https://www.cdc.gov/coronavirus/2019ncov/lab/guidelines-clinical-specimens.html [Accessed on May 26th, 2020] 
[9] Lednicky JA, Lauzardo M, Hugh Fan Z et al. Viable SARS-CoV-2 in the air of a hospital room with COVID-19 patients. Int $\mathrm{j}$ infect Dis 2020; 100: 476-482

[10] Centers for Disease Control and Prevention - Novel Coronavirus (2019-nCoV) Real-time RT-PCR Primers and Probes. https://www.cdc. gov/coronavirus/2019-ncov/lab/rt-pcr-panel-primer-probes.html [Accessed on October 2nd, 2020]

[11] University of Florida home care instructions for people who have or might have COVID-19. https://bridge.ufhealth.org/response/wpcontent/uploads/sites/51/2020/03/3161744-COMM-COVID19_HomeCare-Instructions_FHR_F4.pdf [Accessed on August 29th, 2020]

[12] Guidance for resuming Gl endoscopy and Practice Operations after the COVID-19 Pandemic. https://www.asge.org/docs/default-source/ default-document-library/asge-guidance-for-reopeningl_4-28-2020. pdf [Accessed on September 1st, 2020]

[13] The ACG Endoscopy Resumption Task Force: Guidance on Safely Reopening Your Endoscopy Center. https://webfiles.gi.org/docs/policy/ 2020resuming-endoscopy-fin-05122020.pdf [Accessed on September 1st, 2020]

[14] Tian Y, Rong L, Nian W et al. Gastrointestinal features in COVID-19 and the possibility of faecal transmission. Aliment Pharmacol Ther 2020; 51: 843-851

[15] Forde JJ, Goldberg D, Sussman D et al. Yield and implications of preprocedural COVID-19 PCR testing on routine endoscopic practice. Gastroenterology 2020; 1538: 1538-1540

[16] Bowyer B, Thurkral C, Patel S et al. Outcomes of symptom screening and universal COVID-19 reverse transcriptase polymerase chain reaction testing before endoscopy in a community-based ambulatory surgery center. Gastrointest Endosc 2021; 93: 1060-1064.e1

[17] Podboy A, Cholankeril G, Cianfichi L et al. Implementation and impact of universal pre-procedural testing of patients for COVID-19 prior to endoscopy. Gastroenterology 2020; 17: S0016-5085(20)34819-8 doi:10.1053/j.gastro.2020.06.022

[18] Florida's COVID-19 Data and Surveillance Dashboard for Alachua County. https://experience.arcgis.com/experience/96dd742462124 fa0b38ddedb9b25e429 [Accessed on September 1st, 2020]
[19] West CP, Montori VM, Sampathkumar P. COVID-19 testing: the threat of false-negative results. Mayo Clin Proc 2020; 95: 1127-1129

[20] Fang Y, Zhang H, Xie J et al. Sensitivity of CT for COVID-19; comparison to RT-PCR. Radiology 2020; 296: E115-E117

[21] Wu Y, Guo C, Tang L et al. Prolonged presence of SARS-CoV-2 viral RNA in faecal samples. Lancet Gastroenterol Hepatol 2020; 5: 434435

[22] Xiao F, Tang M, Zheng $X$ et al. Evidence for gastrointestinal infection of SARS-CoV-2. Gastroenterology 2020; 158: 1831-1833

[23] Bonato G, Disocridi L, Mutgnani M. Faecal-oral transmission of SARSCoV-2: practical implications. Gastroenterology 2020: S0016-5085

[24] Grassia R, Testa S, Pan A et al. SARS-CoV-2 and gastrointestinal tract: The dark side of the pandemic. Dig Liver Dis 2020; 52: 700-701

[25] Woloshin S, Patel N, Kesselheim A. False negative tests for SARS-CoV2 infection - challenges and implications. N Engl J Med 2020; 383: e38

[26] Strong JE, Feldmann H. The crux of Ebola diagnostics. J Infect Dis 2017; 216: 1340-1342

[27] Wölfel R, Corman VM, Guggemos W et al. Virological assessment of hospitalized patients with COVID-2019. Nature 2020; 581: 465-469

[28] Bullard J, Dust K, Funk D et al. Predicting infectious severe acute respiratory syndrome coronavirus 2 from diagnostic samples. Clin Infect Dis 2020; 71: 2663-2666

[29] Zheng S, Fan J, Yu F et al. Viral load dynamics and disease severity in patients infected with SARS-CoV-2 in Zhejiang province, China, January-March 2020: retrospective cohort study. BMJ 2020; 369: m1443

[30] Zou L, Ruan F, Huang M et al. SARS-CoV-2 Viral load in upper respiratory specimens of infected patients. N Engl J Med 2020; 19: 11771179

[31] To KK, Tsang O, Leung WS et al. Temporal profiles of viral load in posterior oropharyngeal saliva samples and serum antibody responses during infection by SARS-CoV-2: an observational cohort study. Lancet Infect Dis 2020; 20: 565-574

[32] Byrne AW, McEvoy D, Collins AB et al. Inferred duration of infectious period of SARS-CoV-2: rapid scoping review and analysis of available evidence for asymptomatic and symptomatic COVID-19 cases. BM] Open 2020; 10: e039856 